\title{
La profesión docente en la sociedad del conocimiento. Una mirada a través de la revisión de investigaciones de los últimos 10 años
}

\section{The teaching profession in the knowledge society. A look through the research review of the last 10 years}

\author{
Lourdes Montero \\ Universidad de Santiago de Compostela. Santiago de Compostela (España) \\ lourdes.montero@usc.es \\ Adriana Gewerc \\ Universidad de Santiago de Compostela. Santiago de Compostela (España) \\ adriana.gewerc@usc.es
}

\section{Resumen}

Este artículo tiene el propósito básico de aproximarse a los desafíos que se le plantean a la profesión docente ante las nuevas condiciones sociales, económicas, culturales y tecnológicas del siglo XXI. Lo hacemos mediante la revisión de un conjunto de publicaciones derivadas de investigaciones realizadas por las autoras durante los últimos diez años en el seno del Grupo de investigación Stellae (htttp.stellae.usc.es), para identificar $\mathrm{y}$ analizar resultados que ponen en juego factores macro, meso y micro que articulan las respuestas de profesorado e instituciones concernidas. Recurrimos así mismo a investigaciones y autores que nos ayudan a comprender cuáles son esos desafíos, de dónde proceden, quienes los plantean y qué significados emergen para la profesión de la enseñanza.

Las conclusiones más relevantes muestran cómo se complementan las políticas educativas con la formación inicial para profundizar en la desvalorización del profesorado incentivando su pérdida de autonomía. Con este bagaje el profesorado y las instituciones se dirimen entre la conservación y el cambio, a pesar de las demandas de transformación procedentes del impulso tecnológico vigente.

Palabras clave: Culturas docentes, culturas organizativas, formación y desarrollo profesional docente, profesión docente, Tecnologías digitales.

\footnotetext{
Abstract

This article has the basic purpose of approaching the challenges posed to the teaching profession to face the 21 st century social, economic, cultural and technological conditions. We do this by reviewing a set of publications derived from research carried out by the authors during the last ten years within the Stellae Research Group (httt.stellae.usc.es) to identify and analyze results that put macro meso and micro factors into play, which articulate the answers of faculty and institutions concerned. We also resort to research and authors that help us understand what these challenges are, where they come from, who poses them and what meanings emerge for the teaching profession.

The most relevant conclusions show how the educational policies complement the initial training to deepen the devaluation of the teaching staff, encouraging their loss of autonomy. With this background, faculty and institutions are divided between conservation and change, despite the demands for transformation stemming from the current technological impulse.

Key words

Teacher cultures, organizational cultures, teacher education and professional development, teaching profession, Digital technologies.
} 


\section{Introducción}

Las cuestiones relacionadas con las características y condiciones de la profesión docente son objeto permanente de atención en la investigación sobre educación. El interés en las mismas se justifica en el papel relevante que el profesorado desempeña para la educación y la formación en los distintos niveles y etapas del sistema educativo como mediador de los procesos de enseñanza y aprendizaje. De dónde la preocupación por aproximarse al análisis de la profesión docente cada vez que los cambios sociales, económicos, culturales y tecnológicos fuerzan a reconsiderar el papel del profesorado en la sociedad, de manera que el tema bien puede considerarse como una de esas cuestiones perdurables en educación, una "enduring" cuestión en los contextos cambiantes del mundo en que nos toca vivir (Cochran-Smith, Feiman-Nemser y McIntyre, 2008).

Diversos autores han abordado este tema. Por ejemplo, Hargreaves (1996), en Profesorado, cultura y postmodernidad. Cambian los tiempos, cambian los profesores, plantea un análisis de las paradojas de la postmodernidad y los desafíos para la escuela y los profesores que sintetiza en siete dimensiones fundamentales. Si las reglas del mundo están cambiando, dice, es necesario que los profesores cambien con ellas. Unos años después, Hargreaves (2003) retoma el análisis de los retos que para el profesorado significa Enseñar en la sociedad del conocimiento. Afirma de manera contundente que no habrá sociedad del conocimiento sin docentes de alta capacitación. En simultáneo, califica a la docente de "profesión paradójica" (se espera todo de los docentes y a la vez se les desvaloriza) y pone el dedo en la llaga de las contradicciones de la sociedad del conocimiento y de sus dificultades para hacer de la profesión de la enseñanza una "verdadera profesión del aprendizaje", denominación propuesta por Darling-Hammond y Sykes en 1995 para caracterizar a la profesión docente.

Sin entrar en la profundidad de estos asertos en sus contextos de producción, lo que parece más bien deducirse de los mismos es la llamada de atención hacia el reconocimiento a los saberes y compromisos del profesorado para con las sociedades en las que viven y trabajan, pero también, la corresponsabilidad de las mismas para con el profesorado. Los profesores son las "comadronas" de la sociedad del conocimiento de modo que, sin ellos o sin su competencia profesional, el futuro "nacerá muerto o será deforme" (Goodson y Hargreaves, 2007, p. xi).

Las tecnologías son un factor ineludible a la hora de representar y caracterizar los cambios producidos en el paso de las sociedades industriales a la sociedad global del conocimiento (Fishman y Dede, 2016). Su presencia ha configurado nuevas formas de enseñar y aprender y provocado un sin fin de demandas y expectativas para la escuela y el profesorado ¿Qué condiciones se han dispuesto para afrontarlas?

En este artículo pretendemos explorar algunas respuestas a este desafío, probablemente uno de los imperativos más visibles, a su vez estrechamente relacionado con las condiciones socioeconómicas y culturales de este siglo XXI. Lo hacemos mediante la revisión de un conjunto de publicaciones derivadas de investigaciones realizadas por las autoras durante los últimos diez años en el seno del Grupo de investigación Stellae (htttp.stellae.usc.es) para identificar y analizar resultados que ponen en juego factores

La profesión docente en la sociedad del conocimiento. Una mirada a través de la revisión de investigaciones de los últimos 10 años. Lourdes Montero y Adriana Gewerc. Página 2 de 22 
macro, meso y micro que articulan las respuestas de profesorado e instituciones. Recurrimos así mismo a autores y estudios que posibilitan comprender qué desafíos se plantean y qué significan para la profesión de la enseñanza.

\section{Marco teórico conceptual}

Afrontamos el marco teórico conceptual de referencia mediante el desarrollo de dos sub apartados. En el primero, buscamos situar a la profesión docente en el complejo mundo en el que desarrolla su actividad claramente impactada por las condiciones sociales, políticas, económicas y culturales y la poderosa omnipresencia de las tecnologías. En el segundo, buceamos en las contradicciones emergentes en el doble discurso de desvalorización y revalorización de la escuela y la profesión docente. Ambos se fundamentan en la diversidad de fuentes documentales empleadas para el análisis e interpretación de este artículo.

\subsection{El profesorado ante la sociedad contemporánea}

La revisión de Cochran-Smith y Villegas sobre el estado de la investigación en el campo de la formación del profesorado -inicial e inducción- publicada incompleta en dos artículos de la revista Journal of Teacher Education (2015a, 2015b) y completa en el capítulo titulado "Research on Teacher Preparation: Charting the Landscape of a Sprawling Field", incluido en el Handbook of Research on Teaching, 5th edition (Gitomer \& Bell, 2016), resulta esclarecedora para el análisis de la situación de la profesión docente al configurar un escenario claramente impactado por las condiciones sociales, económicas, políticas, culturales y tecnológicas. Tres argumentos principales estructuran el capítulo: A) El enfoque de la formación del profesorado como una práctica social históricamente situada, una herramienta conceptual efectiva para "domesticar" un campo disperso y denso de investigación. Tres grandes programas de investigación identificados expresivos a su vez de tres tendencias: 1) Investigación sobre rendición de cuentas (accountability), eficacia (calidad docente) y políticas en la formación inicial, que revela una atención sin precedentes; 2) la preparación del profesorado para la sociedad del conocimiento (la tendencia acerca de las concepciones cambiantes de cómo aprenden las personas y qué necesitan conocer, y 3) la preparación del profesorado para la diversidad y la equidad (la tendencia del incremento de la diversidad de las poblaciones estudiantiles y de la desigualdad). Cada uno de estos tres programas incluye "clusters" y líneas de investigación en función de los estudios seleccionados. Transversales a todos ellos, las prácticas de investigación utilizadas.

B) Las relaciones existentes entre las prácticas de las investigaciones y el poder social, económico e institucional, por otro. Para las autoras, hay diferencias en el grado en el que las investigaciones se alinean con la agenda de reforma neoliberal dominante, de manera que a pesar de adoptar el foco de la equidad mantienen las relaciones de poder existentes y así apoyan de forma tácita las desigualdades (estudios en esa dirección y otros desafiándola).

C) El tercer argumento alude a las limitaciones de la revisión y la necesidad de nuevas líneas de investigación: 1) ampliar y profundizar la comprensión de la práctica de la formación y el aprendizaje de los candidatos más allá de un curso, trabajo de campo (prácticas) y un programa, aspectos más atendidos en los estudios revisados. 2) Examinar en el tiempo cómo y bajo qué condiciones los futuros profesores aprenden las

La profesión docente en la sociedad del conocimiento. Una mirada a través de la revisión de investigaciones de los últimos 10 años. Lourdes Montero y Adriana Gewerc. Página 3 de 22 
complejas habilidades (skills) de la enseñanza como prácticos reflexivos (relaciones creencias y prácticas), tanto como aprender a negociar habilidades y prácticas clave y adaptarlas a las diferentes necesidades de los estudiantes en el contexto de las condiciones locales y de culturas de las escuelas. 3) Explorar cuestiones desarrolladas conjuntamente por la escuela y la universidad acerca de las conexiones entre el aprendizaje de los profesores y de los estudiantes, incluyendo las condiciones que apoyan u obstaculizan la construcción de entornos para enriquecer las oportunidades de estudiantes marginados y desafiar tradiciones de bajas expectativas. Y 4) Reclamar más investigación sobre las relaciones de la formación del profesorado y los factores sociales, culturales e institucionales, en particular el impacto de la pobreza sobre la enseñanza, el aprendizaje y la formación del profesorado.

El análisis realizado por Cochran-Smith y Villegas (2016), nos sirve como un marco de referencia para examinar la incidencia de este conjunto de tendencias en el sentido del inter-juego entre la escuela, el profesorado y las tecnologías en la sociedad del conocimiento. Las tres señaladas se interrelacionan y compiten debido a las contradicciones emergentes de sus supuestos sociales, económicos, políticos, culturales y educativos subyacentes.

Uno de los cambios más decisivos es el del tránsito de una economía industrial a una economía del conocimiento, global, que ha acabado colocando en primer lugar el poder de los mercados, fruto del neoliberalismo galopante (capitalismo salvaje, en palabras del recordado profesor Herminio Barreiro, 2005), junto a otros así mismo determinantes, como los demográficos y migratorios. Cambios que no sólo han transformado las sociedades y la vida de las personas, también han focalizado la atención en la calidad de los sistemas educativos y del profesorado, asumidos como principales responsables de la formación de los futuros trabajadores para la nueva economía del conocimiento (Cochran-Smith y Villegas, 2016; Hargreaves, 1996, 2003; OECD, 2005; Subirats, 1999; World Bank, 2010).

Lo que caracteriza a este momento histórico, afirma Sadin (2017), no es sólo la posibilidad de comunicarse en todos los niveles, ni acceder a información insospechada, como pensábamos en los inicios del siglo. Estos hechos son altamente significativos, pero el cambio más trascendental está en dos aspectos: por un lado, en la capacidad de interpretación e iniciativa que está adquiriendo la técnica y, por otro, la "subjetividad ampliada" con posibilidades hasta ahora inéditas y aumentadas por procesos cognitivos superiores.

"La potencia de recopilación y almacenamiento de datos, la sofisticación algorítmica, y la velocidad de almacenamiento permiten a los procesadores evaluar en tiempo real una gran cantidad de parámetros, establecer cartografías precisas de situaciones en curso, sugerir soluciones a entidades humanas, o tomar ellos mismos decisiones, en función tanto de criterios determinados como de factores aleatorios. Es la construcción de una potencia interpretativa y reactiva que caracteriza con exactitud la inteligencia tecnológica contemporánea, en su doble poder dinámico de comprensión y acción.” (Sadin, 2017, p. 66).

El mundo de los datos se vuelve el elemento intangible, ubicado en paralelo, que está mediando nuestra relación con el mundo, y la velocidad óptica de su acceso proporciona al sujeto una asistencia hiper-individualizada con una tecnología que se inmiscuye en la vida cotidiana de manera constante, extendiendo con esto nuestras posibilidades físicas.

La profesión docente en la sociedad del conocimiento. Una mirada a través de la revisión de investigaciones de los últimos 10 años. Lourdes Montero y Adriana Gewerc. Página 4 de 22 
Por lo tanto, mientras en la primera quincena del siglo XXI, se hablaba de sociedad digital, en estos momentos, se están sobrepasando los límites para encontrarnos con un mundo paralelo que cartografía la realidad con datos que son capaces de organizar la vida de las personas y predecir resultados. Así mismo, la tecnología amplía nuestras posibilidades de modo inconcebible en otro tiempo. No es sólo un instrumento, es una extensión de nuestro cuerpo y nuestra cognición.

En ese contexto, vivimos penduleando entre una fe ciega en la tecnología (visión totémica para Sadin), y el rechazo por el miedo a su dominación y control, negando su existencia. Ni una ni otra posición nos ofrece la conciencia del significado profundo de la condición humana en este momento histórico. Será necesario marcar un distanciamiento para conocer qué está sucediendo y cómo son las nuevas reglas que están en juego con una implicación responsable.

Lo cierto es que todos estos cambios que caracterizan la contemporaneidad se desarrollan en contextos concretos y con intereses económicos situados. Los cambios socio-económicos han sido gestionados, de modo dominante, desde una perspectiva neoliberal con consecuencias en las políticas educativas y en las prácticas desarrolladas en las instituciones que las llevan adelante. Son muchos los autores que analizan estas repercusiones y las consecuencias para el trabajo docente o el modo en que se entiende la profesión y su desarrollo profesional. (Véase Apple, 2005; Cochran-Smith y Villegas, 2016; Escudero, 2002, 2003; Fishman y Dede, 2016; Hargreaves, 1996, 2003; Torres Santomé, 2017).

Como consecuencia, han aumentado de forma exponencial las expectativas y demandas sociales dirigidas a la profesión docente y a la formación del profesorado situándolas en un aparente callejón sin salida, uno de los resultados puestos de relieve en nuestros estudios (Zapico, Martínez Piñeiro y Montero, 2017). Entre otras expectativas y demandas, la del mandato dirigido a la consecución de estándares comprobados a través de pruebas de evaluación específicas, un fenómeno que afecta al conjunto del profesorado y resulta particularmente visible para el profesorado y alumnado de las etapas educativas obligatorias. Pero también, la atención a cómo aprenden las personas y qué necesitan conocer junto a atender la diversidad de los estudiantes y a las desigualdades crecientes.

\subsection{El profesorado y la escuela}

El panorama brevemente dibujado arriba ha traído consigo un doble discurso. Por un lado, la necesidad de revalorizar una profesión que tiene en sus manos el futuro de las generaciones venideras. Pero por otro, viene produciéndose en las últimas décadas una desvalorización de su trabajo como profesionales de la enseñanza, reduciendo los márgenes de libertad en la toma de decisiones profesionales y regulando su quehacer cotidiano de forma exhaustiva, a través del aumento de las presiones evaluativas (tanto del alumnado como del profesorado, directa e indirectamente a través del alumnado). Una clara constatación del carácter de "profesión paradójica" evidenciado por Hargreaves (2003). Una aparente contradicción que algunos autores han denominado como "el gran retroceso" (Imbernón, Gimeno, Rodríguez Martínez y Sureda, 2017). El proceso performativo que las políticas neoliberales han planteado, ha ayudado a configurar una mirada social de la profesión y del profesorado pero, al mismo tiempo, ha generado una manera de pensarse a sí mismo como profesionales (Ball, 2012).

La profesión docente en la sociedad del conocimiento. Una mirada a través de la revisión de investigaciones de los últimos 10 años. Lourdes Montero y Adriana Gewerc. Página 5 de 22 
Para Nóvoa $(2009,2011)$, es preciso un mayor protagonismo de los propios profesores y profesoras en la reivindicación de su función y en el refuerzo de su presencia pública aunque ello signifique que la profesión se torne más "vulnerable":

Se habla mucho de las escuelas y de los profesores. Hablan los periodistas, los columnistas, los universitarios, los expertos. No hablan los profesores. Hay una ausencia de los profesores, asistimos a una suerte de silencio de una profesión que ha perdido visibilidad en el espacio público. Hoy día se impone una apertura de los profesores al exterior. Comunicar con la sociedad es también responder ante la sociedad. Posiblemente la profesión se volverá más vulnerable, pero esta es la condición necesaria para afirmar su prestigio y su estatuto social (Nóvoa, 2009, p. 55).

Asistimos, por tanto, a un movimiento que, por un lado, demoniza la profesión docente a través de la crítica sistemática a un sistema educativo, que resulta a-funcional para los nuevos objetivos de las sociedades del conocimiento, que apuntan al talento, la creatividad, el emprendimiento y la capacidad de resolver problemas como los ejes sobre los que pivotar el quehacer de la escuela (entendida en sentido amplio como institución educativa) y el profesorado y, por otro, aumenta las demandas y exigencias a atender, mientras desconfía de las capacidades profesionales de profesores y profesoras, como se pone de manifiesto en diferentes estudios, los nuestros entre otros (Pérez-Díaz y Rodríguez, 2013; Hargreaves, 2003; Zapico, Martínez Piñeiro y Montero, 2017).

Sin embargo, no se toman medidas reales que propicien que la institución educativa avance en el sentido de dar respuestas a estos desafíos; de modo que la "gramática de la escuela" (Tyack y Tobin, 1994) -su estructura más sólida, profundamente arraigadapermanece y se rechaza cualquier desviación que la contradiga -sea o no razonable y adecuada- en la dirección de revertir esa gramática y apuntar a su transformación. Al mismo tiempo, las medidas en pro de la calidad y rendición de cuentas, tampoco apuntan hacia ello. Los docentes se encuentran entonces como parte de un sistema heredado que les encorseta, con normativas que regulan su trabajo en el día a día, con escaso poder de discusión, y la espada de Damocles de la evaluación sobre su eficacia docente pendiendo sobre sus cabezas. Sumisión, desconcierto, perplejidad, aumento del malestar y sálvese quien pueda son consecuencias previsibles de este embrollado estado de cosas. Para Hargreaves (2003), los docentes se encuentran "atrapados" en un triángulo de intereses de modo que sus respuestas ante este estado de cosas pueden situarse en la asunción como víctimas, catalizadores o contrapuntos del desempeño de su papel en la sociedad del conocimiento.

Como hemos señalado en otro lugar (Zapico, Martínez Piñeiro y Montero, 2017), conservación y cambio son ejes que configuran y atraviesan la profesión docente. Y es probablemente por la especificidad de esta paradoja, que ser profesor necesite en cada tiempo y lugar reconsiderar sus significados, de forma que el tema vuelve una y otra vez a la noria de las cuestiones perdurables en la investigación educativa, aquellas que no se resuelven de una vez por todas. El compromiso con una escuela abierta al mundo, situada a la vez en un determinado contexto, interpelada por los cambios sociales y tecnológicos, es una de esas gruesas cuestiones perdurables en educación. Enkvist (2016) recuerda a Arendt, para quien la esencia de la educación es necesariamente conservadora, porque su tarea es transmitir a las generaciones venideras la cultura creada por nuestros antepasados. La autora invita a recomponer la dignidad de la profesión docente recuperando el valor de la enseñanza.

La profesión docente en la sociedad del conocimiento. Una mirada a través de la revisión de investigaciones de los últimos 10 años. Lourdes Montero y Adriana Gewerc. Página 6 de 22 
No obstante, resulta tremendamente complejo hablar de la profesión docente como si ésta fuera un todo. Al hacerlo se corre el riesgo de obviar el mundo personal y profesional de cada profesor y profesora, inmersos en un contexto determinado, con una formación específica, trabajando en instituciones y entornos sociales diversos, con niveles de compromiso diferentes. Reconocer esta realidad implica, en simultáneo, asumir que la profesión docente, como cualquier otra, es una construcción social, histórica, y cada uno de sus miembros representa, por tanto, el juego de los diversos contextos confluyentes (macro, meso y micro). De ahí la posibilidad del estudio de la profesión docente en su globalidad, de sus características específicas como tal, de las culturas que convergen o divergen en su seno, de su evolución, su pasado y su presente futuro. Investigar en este campo significa, por tanto, una atención extremada a este doble juego pues, como se constata en los estudios realizados por las autoras, trabajamos como investigadoras con profesores y profesoras de carne y hueso que están, a su vez signados por su pertenencia a las prácticas sociales de su profesión.

$\mathrm{Y}$ en este presente futuro emerge con fuerza su ubicación en un mundo de conocimientos acelerados donde las tecnologías adquieren un papel disruptivo, al provocar pensamientos y sentimientos contradictorios en relación con su valor como profesionales de la enseñanza, al poner en cuestión los significados de enseñar y "diluir" el papel y el lugar de los profesores. Como si el futuro fuera a concretarse en un profesorado ausente de las aulas porque éstas estarán gobernadas por dispositivos digitales con prestaciones múltiples e insospechadas. Es interesante mencionar que el profesor español propuesto para los "nobel" de educación en 2017 es alguien que enseña a través de la plataforma Youtube, fuera del sistema educativo formal (https://www.youtube.com/watch?v=UDlOJqyJNt0. ¿Asistimos acaso a un proceso de deslegitimación de la educación escolar, de la escuela como institución, ante el poder de las tecnologías? ¿Por qué? ¿Es a ese lugar hacia dónde vamos? Las palabras de Inés Dussel permiten entrever algunas respuestas:

Las nuevas tecnologías tienen lógicas y modos de configurar el conocimiento muy diferente a los de la escuela. Las primeras funcionan en base a la personalización, la seducción y el involucramiento personal y emocional, y suelen ser muy veloces y con una interacción inmediata. La escuela, en cambio, es una institución basada en el conocimiento disciplinar, más estructurada, menos exploratoria, y con tiempos y espacios determinados de antemano, más lentos y menos porosos. Cabe esperar entonces un proceso de negociación y de reacomodamiento de la institución escolar que no será automático ni inmediato, y que no debería ser leído sólo como resistencia al cambio. (Dussel, 2011, p.13).

En 1999 Marina Subirats analizaba el lugar del sistema educativo en las etapas y tránsitos de las sociedades tradicionales (pre siglo XX) a las sociedades industriales del siglo XX y a las postindustriales del XXI. Sus palabras continúan teniendo plena vigencia:

... Aunque podría pensarse en otras formas institucionales, hoy por hoy la única institución expresamente diseñada para la formación de las personas jóvenes, y que ofrece cierta garantía de cobertura universal (aunque no igualitaria) es el sistema educativo. De modo que hay que emprender seriamente un nuevo debate sobre sus funciones, sobre las tareas que socialmente le son encomendadas, para ver cómo,

La profesión docente en la sociedad del conocimiento. Una mirada a través de la revisión de investigaciones de los últimos 10 años. Lourdes Montero y Adriana Gewerc. Página 7 de 22 
paulatinamente, puede reconvertirse hacia una institución no sólo transmisora de conocimientos, sino también transmisora, de modo patente, de criterios y normas de comportamiento (Subirats, 1999, p. 177).

Por un lado, la escuela se enfrenta al reto de acomodarse a otras formas de enseñar y aprender $\mathrm{y}$, por otro, continúa teniendo pleno sentido como una de las pocas instituciones supervivientes con un papel intencional de transformación social. La escuela como un lugar de salvación para tantos pues sin educación no hay futuro. La escuela, afirma Dussel (2016) "debería ser el espacio de lo difícil pero importante". En su trabajo, la autora reflexiona sobre las contradicciones que emergen del choque de dos mundos. La idea de la inmediatez y lo fragmentario enfrentada a la estabilidad que necesitan las aulas para funcionar; las "promesas" de las tecnologías de más fácil, rápido y mejor aprendizaje, como algo mágico, obviando su dependencia de las decisiones y actuaciones de los profesores; del modo como los sujetos y las instituciones procesan los cambios; las escuelas cambian pero lo hacen de forma más lenta, tienen tradiciones muy instituidas; pensar "para qué traemos las tecnologías", qué nos permiten aprender, qué complejidad y desafíos plantean, cuáles son los objetivos educativos, de qué vale la pena apropiarse, $y$, dos cuestiones urgentes: más exploración que navegación sin rumbo; más argumentos fundamentados que opinión. Alude también a las dificultades en función de los curricula, las estructuras horarias, el número de alumnos por aula, para hacer frente a los desafíos provocados por las tecnologías. Identifica y desmonta tres mitos: los nativos digitales ya saben hacer todo; las tecnologías "capturan" la atención de los estudiantes, y el mito de los docentes resistentes a las tecnologías.

A pesar de las críticas y el cuestionamiento, las escuelas son espacios de esperanza, pues sin ella se vuelven lugares imposibles, afirman Murillo y Krisesky (2015):

En ellas se depositan grandes expectativas: se espera que los alumnos aprendan y crezcan como personas, que los docentes innoven y perfeccionen sus prácticas, que se contribuya a la creación de sociedades más justas... Hoy, quizás más que nunca en la historia, son objeto de críticas más y menos informadas, más y menos constructivas. El imperativo de que la escuela debe mejorar la calidad de su propuesta no es una novedad. Sin embargo, las voces que hoy se alzan a favor de una profunda reconversión de la institución escolar son más multifacéticas, impetuosas y lapidarias (Murillo y Krichesky, 2015, p.70).

Profesores y profesoras ejercen la influencia más determinante en el aprendizaje de los alumnos y, por tanto, la atención a la calidad de su formación y de sus condiciones de trabajo debe ser un objetivo político prioritario dirigido a reforzar su compromiso intelectual y emocional con la complejidad de su tarea (Montero, 2006). El profesorado "marca la diferencia", resulta una afirmación claramente aceptada y evidenciada por la investigación educativa, aunque este reconocimiento haya tenido una escasa repercusión en las políticas educativas llevadas a cabo en los últimos años en nuestro entorno, como señalábamos más arriba. Ningún cambio o reforma educativa puede resultar exitosa sin colocar al profesorado en el centro de la ecuación de sus propuestas y expectativas. Pero, más allá de la retórica que con frecuencia esconde esta afirmación, hemos aprendido también que son las culturas y las instituciones las que posibilitan o dificultan marcar esa diferencia. De ahí que el profesorado haya sido el referente principal de

La profesión docente en la sociedad del conocimiento. Una mirada a través de la revisión de investigaciones de los últimos 10 años. Lourdes Montero y Adriana Gewerc. Página 8 de 22 
nuestras investigaciones sobre qué sucede cuando las tecnologías se instalan en las aulas y los centros educativos con promesas de más y mejor en sus actuaciones y resultados (Gewerc y Montero, 2013; Montero y Gewerc, 2013).

Profesores y profesoras trabajan en instituciones llamadas escuelas -desde Infantil a la Universidad-, a las que se incorporan con una formación y cultura determinada fruto de su socialización familiar y escolar, de su condición de futuros profesores en las instituciones de formación inicial y como profesionales más tarde en los diversos tipos de escuela, a los que afectan y por los que son afectados. En el desarrollo de este proceso, las tecnologías han formado parte de su vida, contribuyendo a configurar una cultura acerca de su lugar en los procesos de enseñanza y aprendizaje. La literatura educativa es un buen exponente de la importancia de las mismas adoptando, según el momento histórico, diferentes énfasis para situar su lugar en el proceso educativo de la mano del profesorado deseablemente preparado para su uso. Y si esto ha sucedido con una cierta parsimonia durante etapas anteriores, la sociedad del conocimiento ha supuesto una aceleración sin precedentes, tanto por el desarrollo científico tecnológico de los dispositivos disponibles como por los cambios producidos en las relaciones sociales, en las interacciones con otros, en la emergencia de las redes sociales, la enseñanza y el aprendizaje (Fishman y Dede, 2016). ¿Qué sucede cuando las tecnologías irrumpen en las escuelas?

La actividad de enseñar es un trabajo complejo, con una complejidad aumentada y expandida en los tiempos que corren que exige más y más de la competencia profesional del profesorado $\mathrm{y}$, en consecuencia, de la consideración social y política de su importancia. Sin embargo, como estamos mostrando, a menudo los cambios se promueven sin contar con su colaboración e incluso a pesar de la ausencia de su compromiso profesional con ellos.

$\mathrm{Si}$, como afirman Goodson y Hargreaves (2007) los profesores son las "comadronas" de la sociedad del conocimiento, el corolario sería fortalecer el papel de la escuela y el profesorado en el proceso de enseñar y aprender de niños, jóvenes y adultos. Sin embargo, parece haber una tendencia a dimitir del mismo, como si resultara vergonzoso reconocer que su actividad profesional es la enseñanza. ¿Qué etiquetas hacen que la enseñanza sea una actividad de la que parece hay que dimitir ante el poderío de las tecnologías? ¿Desaparece la enseñanza a favor del aprendizaje como si éste no necesitara de la concurrencia de aquella? Paradójicamente en apariencia, el discurso de los modelos centrados en el aprendizaje parece haber contribuido al fenómeno de desvalorización del profesorado, al que nos referíamos en párrafos anteriores. Conceptos como los de guía, facilitador, orientador, llevan tiempo planteándose como opciones alternativas para definir la actividad docente, cuando son modos de representar la actividad de enseñar no contradictorios con ella. De dónde el interés de algunos autores por resituar el lugar del profesorado. Para Biesta (2017) la enseñanza es esencial y tiene un significado que va mucho más allá de facilitar el aprendizaje y el profesor es alguien que tiene algo que decir y aportar, como reclama Enkvist (2016). Es importante que la escuela retome su función de transmisión y archivo de la cultura, dice Dussel (2011, p. 27), quien alerta al mismo tiempo sobre los problemas de selección y de construcción de repertorios visuales y escritos ante las posibilidades tecnológicas para archivar contenidos.

La escuela ha perdido glamur (si es que lo tuvo alguna vez). También la profesión docente ha perdido atractivo aunque no parezca notarse ante el número de candidatos que acceden a la formación inicial en el contexto español. En definitiva, la pérdida de

La profesión docente en la sociedad del conocimiento. Una mirada a través de la revisión de investigaciones de los últimos 10 años. Lourdes Montero y Adriana Gewerc. Página 9 de 22 
fuerza y efectividad y, a la larga, el fracaso de los cambios que ignoran la complejidad de ser profesor:

"What marks teachers out as good, o better than good, is not only their content knowledge and pedagogical skills. It is their commiment to their teaching, their students and their learning and achievement. Yet, although it is widely acknowledged that commiment and resilience are indispendable to high quality teaching, we do not know much about how these are sustained or not sustained, in times of change" (Day, Sammons, Stobart, Kington \& Quing Gu, 2007, p.2).

La centralidad de los procesos de enseñanza y aprendizaje, colaboración docente y trabajo en redes, implicación de la comunidad, liderazgo sistémico, el debate entre la rendición de cuentas y la responsabilidad, y las nuevas relaciones entre la administración pública y las escuelas, son seis factores o condiciones para la mejora escolar extraídos de las lecciones aprendidas tras 50 años de historia del movimiento de "Mejora de la escuela" (Murillo y Krichesky, 2015).

\section{Metodología}

Como hemos anticipado en la introducción, elaborar este artículo ha supuesto llevar a cabo una "segunda mirada" a los estudios realizados por las autoras en estos últimos diez años sobre los desafíos que las condiciones sociales, económicas, culturales y, en especial, el enorme impacto que los cambios tecnológicos han supuesto para escuelas y profesores, como hemos tratado de mostrar en los apartados anteriores. Hemos llevado a cabo esta "segunda mirada" mediante la revisión de las publicaciones derivadas de estos estudios. En la tabla siguiente, se recogen los proyectos realizados y las publicaciones resultantes de los mismos.

\begin{tabular}{|c|c|}
\hline Proyectos & Publicaciones derivadas \\
\hline $\begin{array}{l}\text { “Estudio de casos de proyectos de } \\
\text { innovación educativa mediados por TIC en } \\
\text { centros educativos de Primaria y } \\
\text { Secundaria", (Referencia SEJ2005-08656), } \\
\text { financiado por el MEC en su convocatoria } \\
\text { del Plan Nacional de I+D+i 2005-08 } \\
\text { (23.000€) y "Proyectos de innovación } \\
\text { educativa mediados por TIC: diseño, análisis } \\
\text { y evaluación de entornos de enseñanza y } \\
\text { aprendizaje", } \\
\text { PGIDIT06PXIB214192PR) financiado por la } \\
\text { Xunta de Galicia (2006-2009). }\end{array}$ & $\begin{array}{l}\text { 1. Montero, L. y Gewerc, A. (Eds.) (2013). Una } \\
\text { historia, cuatro historias. Acompañar proyectos } \\
\text { de innovación educativa con las TIC. Barcelona: } \\
\text { Graó. ISBN: 978-84-9980-522-1. } \\
\text { 2. Zapico, Ma H., Montero, L. y Gewerc, A. (2011). } \\
\text { The collective construction of a school web } \\
\text { portal. An experience of sustainable innovation. } \\
\text { In I. Candel; L. Gómez Chova \& A. López } \\
\text { Martinez (Eds). 4th International Conference of } \\
\text { Education, Research and Innovation (ICERI) } \\
\text { 2011. Madrid (publicado en DVD ISBN: 978-84- } \\
\text { 615-3324-4) } \\
\text { Montero, L. y Gewerc, A. (2013). Enseñanza con } \\
\text { Tecnologías. Un espejo y cuatro miradas } \\
\text { reflejadas. En L. Montero, L. y A. Gewerc } \\
\text { (coords.). Una Historia, cuatro Historias. } \\
\text { Acompañar proyectos de innovación con las TIC. } \\
\text { Barcelona: Graó, pp. 9-27. ISBN: 978-84-9980- } \\
\text { 522-1.281 } \\
\text { Montero, L.; Gewerc, A. y Zapico, H. (2013). De } \\
\text { lo analógico a lo digital. La innovación con TIC } \\
\text { en un centro rural agrupado. En L. Montero y A. } \\
\text { Gewerc, A. (coords.). Una Historia, cuatro }\end{array}$ \\
\hline
\end{tabular}

La profesión docente en la sociedad del conocimiento. Una mirada a través de la revisión de investigaciones de los últimos 10 años. Lourdes Montero y Adriana Gewerc. Página 10 de 22 


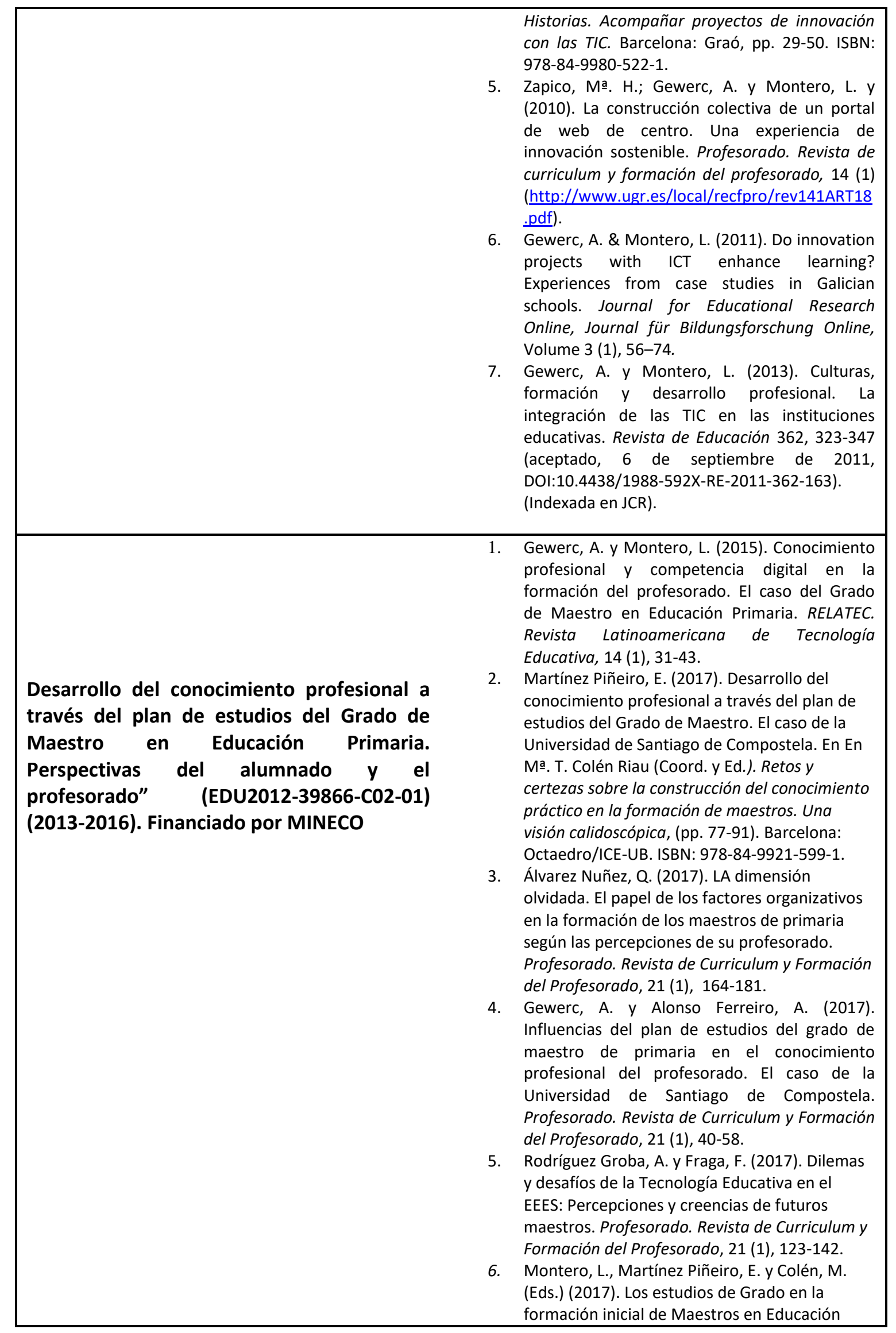

La profesión docente en la sociedad del conocimiento. Una mirada a través de la revisión de investigaciones de los últimos 10 años. Lourdes Montero y Adriana Gewerc. Página 11 de 22 


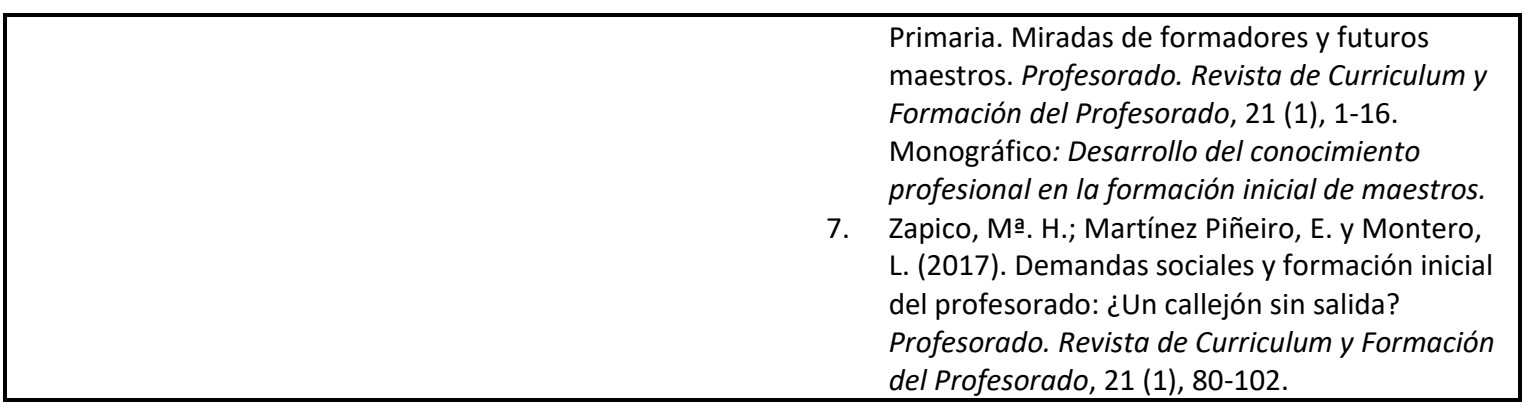

Tabla 1: Proyectos y publicaciones derivadas (elaboración propia)

De la revisión de las publicaciones emergen dos grandes categorías temáticas inclusivas de los diversos resultados obtenidos: Formación inicial y cultura institucional y profesional del profesorado. La primera, extraída fundamentalmente de las publicaciones sobre los resultados del estudio sobre las percepciones de futuros profesores y formadores sobre el conocimiento profesional desarrollado durante su preparación como tales. La segunda, vinculada más específicamente a las publicaciones que dan cuenta de los resultados del proyecto PIETIC, en los que se pone claramente de manifiesto las dificultades que centros y profesores tienen para atender los desafíos tecnológicos del siglo XXI que requieren cambios en la cultura institucional y profesional de gran calado.

Las señas de identidad metodológica de los estudios que originan las publicaciones revisadas han respondido a un enfoque cualitativo en la aproximación a los problemas, en las técnicas y herramientas utilizadas y en la elección de los marcos y herramientas de análisis e interpretación de datos. La opción se justifica en cada caso por el interés puesto en las miradas de los sujetos - profesorado y escuelas- tratando de ese modo de acercarnos al objeto de estudio en su globalidad desde ángulos distintos y colectivos diferentes, contextos institucionales y socio culturales.

La estrategia de investigación ha sido el estudio de casos, por su carácter de respeto a los contextos de producción del conocimiento y las posibilidades de profundización en los problemas. Estudiamos la singularidad y complejidad de un caso determinado para comprender su actividad en circunstancias importantes, como señala Stake (1998). Contribuye a iluminar las complejas e indeterminadas interrelaciones entre la vida y el trabajo de los profesores, el contexto escolar y las iniciativas de cambio y reforma y aproximarnos así a la intensidad emocional de la actividad de enseñar y aprender (Goodson y Hargreaves, 2007).

Los estudios han tenido en común la elección de un estudio de casos múltiple. En los dos estudios PIETIC (Proyectos de investigación educativa con TIC), la estrategia elegida fue la investigación acción colaborativa (I/A) para acompañar, durante un tiempo dilatado, proyectos de innovación con tecnologías desde dentro de la institución y junto al profesorado responsable. El realizado sobre las percepciones de futuros profesores y formadores sobre la construcción del conocimiento profesional a través del desarrollo del plan de estudios del grado de maestro en educación primaria, se ha desarrollado en la Universidad de Santiago de Compostela, a la que pertenecemos. En todos, se emplearon distintas técnicas de obtención de datos y fuentes de información (Yin, 2003), aquellas estimadas más pertinentes en relación con el problema, interrogantes, objetivos y etapas de la investigación. Notas de campo, informes, entrevistas, observaciones, grabaciones en audio y vídeo, fotografías. entrevistas en

La profesión docente en la sociedad del conocimiento. Una mirada a través de la revisión de investigaciones de los últimos 10 años. Lourdes Montero y Adriana Gewerc. Página 12 de 22 
profundidad, relatos y grupos de discusión, son algunas de las técnicas y herramientas más utilizadas.

Destacar, así mismo, que nuestro foco ha estado permanentemente en el sistema educativo formal, con el énfasis en escuelas, futuros profesores, formadores, profesores en ejercicio y miembros de los equipos directivos, como sujetos recurrentes de nuestra trayectoria investigadora.

\section{Discusión y Resultados}

Las investigaciones que hemos realizado en estos últimos 10 años ofrecen un diagnóstico del modo en que el profesorado y la institución en donde desarrolla su trabajo se enfrentan a las condiciones de este momento histórico. En este apartado haremos una síntesis de los principales hallazgos obtenidos mediante la atención a dos grandes categorías inclusivas emergentes de la revisión de las publicaciones seleccionadas. En primer lugar, la formación inicial y las condiciones en que se desarrolla para apoyar a los futuros profesionales a hacer frente a las demandas cruzadas de este primer cuarto del siglo XXI. Y, por otro, la cultura institucional de los centros educativos en donde el profesorado desarrolla su práctica, y la cultura profesional que es construida en esos contextos.

\subsection{Formación inicial}

La investigación precedente muestra la pertinencia y relevancia del periodo de formación inicial, de modo que la ausencia de una preparación inicial formal o el escaso cuidado en su desarrollo curricular e institucional deja a los futuros profesores sin el acceso a los dispositivos básicos para enfrentarse al complejo mundo laboral y continuar su formación y desarrollo profesional (Esteve, 2006; Hudson, Zgaga y Astrand, 2010; Imbernón, 1998, 2017; Montero, 2006, 2008; Cochran-Smith y Villegas 2016; CochranSmith y Zeichner, 2005; Zeichner y Conklin, 2008). De ahí que se le considere un tiempo y un espacio privilegiado para transformar las nociones previas de la socialización primaria y secundaria procedentes de la familia, de la escuela y del largo aprendizaje de la observación de otros profesores, y realizar un complejo cambio conceptual desde el sentido común a puntos de vista profesionales sobre la enseñanza (Feiman-Nemser y Buchman, 1986). Las nuevas condiciones socio-económicas y las demandas que se traducen de ello, requirieron repensar la formación inicial de tal modo que se incluyan contenidos y metodologías que posibiliten al nuevo profesorado enfrentarse a estas nuevas condiciones, de manera crítica y reflexiva, recomendada en otros momentos (por ej. Schön, 1992) y reclamada de nuevo (Cochran-Smith y Villegas, 2016).

En este sentido, los resultados del estudio que se propuso identificar, analizar e interpretar las percepciones de futuros profesores y sus formadores sobre los conocimientos que están construyendo en el desarrollo del plan de estudios del grado de maestro en educación primaria y su contribución a configurar una identidad profesional determinada (Álvarez, 2017; Gewerc y Alonso Ferreiro, 2017; Montero, Colén y Martínez Piñeiro, 2017; Rodríguez Groba y Fraga, 2017), enmarcada en el contexto de la Universidad de Santiago de Compostela, muestra algunas características en las que se concreta el documento del plan de estudios para el futuro profesorado de primaria. Y también la percepción sobre la formación y los conocimientos desarrollados durante ese

La profesión docente en la sociedad del conocimiento. Una mirada a través de la revisión de investigaciones de los últimos 10 años. Lourdes Montero y Adriana Gewerc. Página 13 de 22 
período que tienen tanto el alumnado (futuro docente) como el profesorado (formador de formadores).

El análisis de contenido de los documentos formalizados en el Plan de estudios de Grado de Maestro en Educación primaria de la Universidad de Santiago de Compostela muestra cómo esta universidad ha concretado las demandas proveniente del Espacio Europeo de Educación Superior (EEES), y cómo específicamente ha utilizado los márgenes de acción que las directrices de la Orden ECI/3857/2007, del 27 de diciembre, ha estipulado para esta profesión regulada por el estado.

En principio, anunciar que el viraje hacia competencias de los nuevos planes de estudio (90 competencias) no evidencia cambios significativos en la manera en que el profesorado formador percibe la formación. Tanto alumnado como profesorado perciben que sólo han cambiado las condiciones formales, en cuanto a secuencia, nombre de la materia, o su ubicación en el plan de estudios. Entonces... ¿En qué medida el plan da respuestas a las demandas provenientes del nuevo milenio para con el profesorado? Es decir, para afrontar la complejidad del trabajo del profesorado debido al aumento de los recursos con la llegada de las tecnologías de la información y la comunicación a las aulas; para mejorar los resultados académicos y el peso de la evaluación sistemática a sus espaldas; además de la necesaria atención a la diversidad y la inclusión en todas sus facetas y la mirada hacia lo social para cubrir y alertar cuestiones que, a veces, son desatendidas por la sociedad y las familias, entre otros aspectos.

Las demandas del EEES reclaman mayor coordinación y centralidad en las necesidades formativas del alumnado. Esas nuevas condiciones suponen un profesorado autónomo, reflexivo, capaz de tomar decisiones fundadas y de navegar en los mares de incertidumbre que se imponen en este primer cuarto de siglo. Sin embargo, los diferentes interlocutores tienen una visión fragmentada de la formación inicial, tanto en la dicotomía teoría y práctica, mencionada también por la mayor parte de los investigadores que nos preceden (Flores, 2000; Hauge, 2000; Hobson y Tomlinson, 2001), como en la organización por asignaturas sin coordinación y en la propia visión del conocimiento por los formadores, que persisten en mirarlo bajo el cisma entre lo "pedagógico" y el "contenido" (Gewerc y Alonso Ferreiro, 2017).

En la tensión entre un profesorado autónomo o el que ejecuta decisiones tomadas por otros, vence esta última perspectiva. En su trayectoria formativa, el futuro profesorado no toma decisiones ni asume las consecuencias, sino que sigue el camino marcado desde el inicio, cumpliendo en cada paso los pre-requisitos que le imponen para llegar al objetivo. Esta obediencia es el precio que paga para la obtención de la acreditación correspondiente que le hace merecedor al título de Grado de Maestro en Educación Primaria, necesario para la prueba de oposición y el acceso a la profesión docente. Por otro lado, unos inicios "básicos", alejados de la práctica profesional, le llevan a percibir la "teoría" como algo abstracto, alejado de la realidad, a la que no se puede recurrir para encontrar respuestas. Y limitar la práctica a la aplicación, alejándose del modelo de reflexión sobre la práctica (Schön, 1992), que requiere teoría y teorizar sobre la experiencia.

Con relación a la adaptación a los nuevos entornos generados por las tecnologías, el plan propone seis competencias relacionadas directa o indirectamente con el abordaje de la alfabetización digital -informacional siguiendo el planteamiento de las alfabetizaciones múltiples- (Area, 2005, 2010). Estas competencias son:

La profesión docente en la sociedad del conocimiento. Una mirada a través de la revisión de investigaciones de los últimos 10 años. Lourdes Montero y Adriana Gewerc. Página 14 de 22 
G6: Asumir que el ejercicio de la función docente ha de ir perfeccionándose y adaptándose a los cambios científicos, pedagógicos y sociales a lo largo de la vida.

G11: Conocer y aplicar en las aulas las tecnologías de la información y de la comunicación. Discernir selectivamente la información audiovisual que contribuya a los aprendizajes, a la formación cívica y a la riqueza cultural.

T3: Conocimiento instrumental de las tecnologías de la información y de la comunicación.

T4: La competencia informacional.

E23: Analizar e incorporar de forma crítica las cuestiones más relevantes de la sociedad actual que afectan a la educación familiar y escolar: impacto social y educativo de los lenguajes audiovisuales y de las pantallas; cambios en las relaciones de género e inter generacionales; multiculturalidad e interculturalidad; discriminación e inclusión social y desarrollo sostenible.

E29: Reconocer la mutua influencia entre ciencia, sociedad y desarrollo tecnológico, así como las conductas ciudadanas pertinentes, para procurar un futuro sostenible.

La mayor parte de estas competencias se desarrolla en asignaturas de primer curso, mientras su dedicación va decayendo a medida que se avanza en la carrera. Con la única excepción de la competencia G11 que se mantiene en las fichas de varias asignaturas de todos los años y que incluyen 10 materias en el cuarto curso. Sin embargo, si bien todas las materias del plan de estudios incluyen entre sus competencias la T3 (Conocimiento instrumental de las TIC), está desaparecida en las representaciones de los formadores (Fraga-Varela y Rodríguez-Groba, 2016; Gewerc y Montero, 2015).

A la luz de la complejidad de las expectativas y demandas de la sociedad del conocimiento, parece pues pertinente continuar preguntándose qué deberían saber los futuros profesores para afrontar los desafíos por venir. La formación inicial del profesorado no debería diseñarse y desarrollarse de espaldas al conocimiento de las características y condiciones de la profesión docente a la que aspiran a acceder y de la que algún día serán miembros activos. Profesores y profesoras entran a formar parte de una historia y una cultura previas que les configura y que, deseablemente, contribuirán a configurar.

\subsection{Cultura institucional y profesional del profesorado}

El análisis de las publicaciones sobre los resultados de los estudios que abordaron la problemática de la incorporación de las tecnologías en los centros educativos, con la intención de hacer visibles las relaciones entre los factores personales, profesionales, institucionales y de liderazgo para la innovación sostenible con tecnologías, evidencian la transformación de las culturas profesionales e institucionales ante situaciones de cambio como las que tiene que afrontar el profesorado en este momento histórico.

Como hemos puesto de manifiesto en algunas de esas publicaciones (Gewerc y Montero, 2013; Montero y Gewerc, 2013), cualquier propuesta de transformación de las prácticas de los profesionales de la educación requiere considerar la cultura organizativa en la que se asienta y la cultura profesional del profesorado encargado de hacerlo realidad, ambas estrechamente interrelacionadas.

Bajo el amplio paraguas conceptual de la palabra cultura se incluyen conocimientos, creencias, valores, normas, rutinas y actitudes del profesorado sobre la enseñanza, sobre enseñanza y tecnologías, sobre el alumnado, las relaciones entre profesores, las visiones de su actividad profesional, sus percepciones sobre el cambio, etc. La clave del cambio

La profesión docente en la sociedad del conocimiento. Una mirada a través de la revisión de investigaciones de los últimos 10 años. Lourdes Montero y Adriana Gewerc. Página 15 de 22 
es el significado que éste tenga para los docentes (Gather Thurler, 2004). La cultura, cual Dios Jano, presenta una doble cara. Funciona como marco de referencia con el que interpretar los asuntos profesionales y actuar de modo "apropiado" a cada situación y, en simultáneo, da cuenta de los conflictos y divergencias presentes en las relaciones profesionales. Profesores y profesoras comparten ese marco de referencia cultural propio de una profesión que está, como otras, social e históricamente construida, pero, a su vez, tienen orígenes sociales, edades, experiencias y habilidades distintos.

Sería aventurado afirmar que los profesores sujetos de nuestros estudios comparten una cultura común. Hemos identificado, más bien, una variedad de culturas e identidades a la hora de ejercer la profesión, en función bien de la etapa educativa -Educación infantil, primaria, secundaria- bien del ámbito disciplinar -matemáticas, lenguas, ciencias, educación física, ciencias sociales, etc.- y también del tipo de relaciones que establecen con sus colegas -individualismo, reinos de taifas, colegialidad artificial, colaboración, mosaico móvil- siguiendo la tipología propuesta por Hargreaves (1996).

Así mismo, hemos tenido ocasión de analizar las culturas de los centros en y con los que hemos trabajado, a través de la observación de su actividad, de las rutinas y rituales empleados: distribución de horarios y espacios, usos de espacios e infraestructuras, normas explícitas e implícitas, curriculum oculto. El concepto de cultura se asemeja al de "gramática escolar" (Tyack y Tobin, 1994), al incluir el conjunto de tradiciones y regularidades institucionales sedimentadas a lo largo del tiempo y transmitidas de generación en generación por maestros, profesores y equipos directivos.

El trabajo realizado en las investigaciones evidencia el modo como las políticas regulan el trabajo docente y su consideración como profesionales a través de normativas e instrucciones que en ocasiones se contradicen y que no dejan mucho margen para la autonomía ni para la innovación (aunque haya un mandato explícito hacia ello). Por ejemplo, se envía una pizarra digital por escuela con el mandato de la innovación pero, por un lado, se distribuye la misma cantidad a todos los centros, sin importar el número de aulas, de alumnos, ni las necesidades básicas no resueltas de algunos edificios, poniendo de manifiesto una concepción burocrática de la gestión, Por otro lado, no se apoya con formación, ayuda y mantenimiento, ni se posibilita que exista flexibilidad curricular para el uso de unas herramientas que tienen un lenguaje diferente al dominante. Esta visión también empaqueta medidas sin pensar en los proyectos de los centros, su autonomía, ni la del profesorado que está involucrado personal y profesionalmente con ellos. Al mismo tiempo que se presiona con las evaluaciones externas generando una especie de carrera de obstáculos para lograr no quedar mal parado en las pruebas. Este conjunto de cuestiones ha provocado que, en la mayoría de los centros, los recursos tecnológicos pierdan sentido o estén infrautilizados. Se produce una asociación de la innovación con la incorporación de herramientas tecnológicas en las escuelas, atribuyéndolas un potencial innovador por sí mismas, olvidando que son los contextos y los profesionales los que confieren sentido.

Este discurso contradictorio ayuda al mantenimiento del statu quo, ya que la organización es un artefacto cultural, no solo una estructura, y las dimensiones más invisibles y determinantes de la vida de un centro educativo vienen configuradas por cómo las personas construyen y hacen las organizaciones (González González, 1992:7195; Sosa y Valverde, 2017).

Se ha constatado también la existencia de una interacción permanente entre las culturas del profesorado y las de los centros, alimentándose la una a la otra para conformar un entramado de conservación y cambio. El profesorado no es un agente libre y el modo en

La profesión docente en la sociedad del conocimiento. Una mirada a través de la revisión de investigaciones de los últimos 10 años. Lourdes Montero y Adriana Gewerc. Página 16 de 22 
que encara los cambios derivados del uso de las tecnologías depende de las estrechas relaciones entre los contextos institucionales, culturales y sociales en los que vive y trabaja (Somekh, 2008). La cultura se configura así como factor mediador entre los componentes estructurales y los individuales y grupales. De ahí que identificamos un cruce de culturas entre profesorado y escuela sobre todo cuando se produce alguna alteración a la rutina cotidiana como es el caso de las propuestas de integración de tecnologías en las aulas. En esa instancia se pone en juego la realineación de grupos y subgrupos, lo que moviliza situaciones micropolíticas de lucha por el poder (Gewerc y Montero, 2011, 2013; Montero y Gewerc, 2013). Otro aspecto significativo hace alusión a las creencias que el profesorado tiene sobre su propia capacidad o su eficacia con la tecnología, ya que podría ser un predictor de las posibilidades de integración de la tecnología en el aula (Fraga-Varela, 2014; Rodríguez-Groba y Fraga-Varela, 2017; Wozney, Venkatesh y Abrami, 2006).

El lugar que ocupa la formación para afrontar los retos inscritos en la utilización de tecnologías ha sido una y otra vez destacado, si bien a veces da la impresión de una visión de la formación como algo receptivo que otros "dan". Resulta más difícil ver las enormes posibilidades formativas inscritas en el trabajo de reunirse, intercambiar experiencias y problemas, reflexionar sobre ellos, buscar soluciones, experimentar nuevas herramientas, abrirse a otras ideas, discrepar y profundizar debido quizás al modelo individualista de formación y desarrollo profesional predominante, con frecuencia ajeno al saber experiencial de los profesores.

Cabe aludir también al papel relevante atribuido a la reflexión sobre la práctica en el diseño del estudio de PIETIC a través de la investigación-acción colaborativa. Sin embargo, los resultados muestran una postura de resistencia ante ella. La cultura profesional del profesorado está, en cierta medida, "enfrentada" a la reflexión, de modo que ésta es vivida como una pérdida de tiempo, o una actividad que concierne sólo a los investigadores de la universidad, que tienen el mandato de construir conocimiento y disponen de tiempos y espacios para ello, lo que no sucede en otros niveles educativos (Gewerc y Montero, 2013).

\section{A modo de conclusiones}

El objetivo propuesto para este texto ha sido el de aproximarse a los desafíos que se le plantean a la profesión docente ante las nuevas condiciones sociales, económicas, culturales y tecnológicas del siglo XXI. Hemos abordado este objetivo mediante, por un lado, la revisión de un conjunto de publicaciones derivadas de investigaciones realizadas por las autoras durante los últimos diez años en el seno del Grupo de investigación Stellae (htttp.stellae.usc.es) para identificar y analizar resultados que ponen en juego factores macro, meso y micro que articulan las respuestas de profesorado e instituciones concernidas. Recurrimos así mismo a investigaciones y autores que nos ayudan a comprender cuáles son esos desafíos, de dónde proceden, quienes los plantean y qué significados emergen para la profesión de la enseñanza.

La perspectiva del trabajo pone foco en el sentido del juego entre la escuela, el profesorado y las tecnologías en la sociedad del conocimiento (de la ignorancia la denomina Innerarity, 2015, ante la sobreinformación resultante). Hacerlo de este modo es una necesidad ineludible para comprender con mayor profundidad las situaciones reales y concretas en donde el profesorado construye y desarrolla su identidad profesional. Éste es un momento histórico convulso, gestionado desde una perspectiva

La profesión docente en la sociedad del conocimiento. Una mirada a través de la revisión de investigaciones de los últimos 10 años. Lourdes Montero y Adriana Gewerc. Página 17 de 22 
neoliberal y en el que las tecnologías y los datos que generan, se han vuelto corpóreos y han cambiado la manera en que vivimos y concebimos el mundo, generando nuevas demandas para con la profesión docente y con la escuela. Una sociedad "hiperconectada" en la que el exceso de información exige de las escuelas mantener saberes fundamentales, e impulsar nuevos aprendizajes para responder a los complejos y tremendo desafíos de nuestro tiempo (Innerarity, 2015).

Nuestras investigaciones muestran la necesidad de analizar la profesión docente y su cultura profesional en contexto, identificando las condiciones que la regulan desde las políticas y, al mismo tiempo, observando dónde se desarrolla: la institución, sin perder de vista la decisiva importancia del profesorado. Es decir, el inter-juego entre los niveles macro, meso y micro contaminándose mutuamente.

Los datos evidencian múltiples demandas de nivel macro que se transmutan en políticas que pretenden regular el trabajo cotidiano y muestran cómo las culturas involucradas se movilizan frente a esto. Hemos definido a las demandas como contradictorias y cruzadas, ya que intentan proponer innovaciones, pero al mismo tiempo se legisla o no legisla para limitarlas al tiempo que exigen calidad, responsabilidad y rendición de cuentas.

La formación inicial docente también se ha ido modificando para responder a las demandas procedentes de las condiciones socio-económicas y culturales. En ese sentido, los planes de estudio deberían dar cuenta de contenidos y metodologías que respondan a las concepciones cambiantes de cómo aprenden las personas y qué necesitan conocer en este momento histórico. Hemos visto una muestra que evidencia un escaso cambio en la estructura de los planes. En conjunto, las políticas educativas para con la escuela y la profesión docente y la formación inicial, están ayudando a la desvalorización de su trabajo como profesionales de la enseñanza, reduciendo los márgenes de libertad en la toma de decisiones y regulando su quehacer cotidiano de forma exhaustiva.

En el nivel meso, las culturas profesionales e institucionales se movilizan ante la demanda de cambios, pero de tal forma que generan subgrupos de poder que son portadores o refractores de los cambios en función de su contenido.

$\mathrm{Y}$ es en el cesto construido con todos estos mimbres donde el profesorado está activamente construyendo su identidad profesional.

Presentación del artículo: 20 de Diciembre de 2017 Fecha de aprobación: 20 de enero de 2018

Fecha de publicación: 31 de enero de 2018

Montero, L. y Gewerc, A. (2018). La profesión docente en la sociedad del conocimiento. Una mirada a través de la revisión de investigaciones de los últimos 10 años. RED. Revista de Educación a Distancia, 56. Consultado el (dd/mm/aaaa) en http://www.um.es/ead/red/56/

\section{Financiación}

Esta investigación no ha recibido ninguna subvención específica de los organismos de financiación en los sectores públicos, comerciales o sin fines de lucro.

La profesión docente en la sociedad del conocimiento. Una mirada a través de la revisión de investigaciones de los últimos 10 años. Lourdes Montero y Adriana Gewerc. Página 18 de 22 


\section{Referencias bibliográficas}

Álvarez Nuñez, Q. (2017). ). La dimensión olvidada. El papel de los factores organizativos en la formación de los maestros de primaria según las percepciones de su profesorado. Profesorado. Revista de Curriculum y Formación del profesorado, 21 (1), 164-181.

Apple, M. (2005). Educating the "right" way: Market, standards, God, and inequality. New York, New York: Routledge.

Area, M. (2005). La educación en el laberinto tecnológico. De la escritura a las máquinas digitales. Barcelona: Octaedro.

Area, M. (2010). Tecnologías digitales, multialfabetización y bibliotecas en la escuela del siglo XXI. Boletín de la Asociación Andaluza de Bibliotecarios, n. ${ }^{\circ}$ 98-99, enero-junio, 39-52. dehttp://www.aab.es/aab/images/stories/Boletín/98_99/2.pdf

Recuperado

Ball, S. (2012). The making of a neoliberal academic. Research in Secondary Education 2(1): 29-31.

Barreiro, H. (2005). Prólogo. Junto a las aguas del Atlántico. En M. Area. La educación en el laberinto tecnológico. De la escritura a las máquinas digitales (pp. 13-15). Barcelona: Octaedro.

Biesta (2017). El bello riesgo de educar. Cada acto educativo es singular y abierto a lo imprevisto. España: Ediciones SM.

Cochran-Smith, M. y Villegas, A. (2015a). Framing Teacher Preparation Research: An Overview of the Field, Part 1. Journal of Teacher Education, 66 (1) 7- 20

Cochran-Smith, M. y Villegas, A. (2015b). Critiquing Teacher Preparation Research: An Overview of the Field, Part II. Journal of Teacher Education, 66 (2) 109121

Cochran-Smith, M. y Villegas, A. (2016). Research on Teacher Preparation: Charting the Landscape of a Sprawling Field. In Gitomer, D., \& Bell, C. (Eds.). Handbook of Research on Teaching. 5th Edition (pp. 439-547). Washington DC: American Educational Research Association.

Cochran-Smith, M. \& Zeichner, K. (2005). Studying teacher education: the report of the AERA panel on research and teacher education. New York: Routledge.

Darlig-Hammond, L. \& Sykes, G. (1995). Teaching as the learning profession. Handbook of policy and practice. San Francisco: Yossey-Bas.

Day, Ch.; Sammons, P.; Stobart, G.; Kington, A. \& Gu,Q. ( 2007). Teachers Matter. Conecting Lives, Work and Effectiveness. Mc-Graw Hill Education: Berkshire (England).

Dussel, I. (2011). Aprender y enseñar en la cultura digital. En VII Foro Latinoamericano de Educación. Experiencias y aplicaciones en el aula. Aprender y enseñar con nuevas tecnologías. Buenos Aires: Fundación Santillana.

Dussel, I. (2016). Entrevista en La Nación. Recuperado de http://www.lanacion.com.ar/1929399-ines-dussel-la-escuela-deberia-ser-elespacio-de-lo-dificil-pero-importante

Enkvist, I. (2016). El complejo oficio del profesor. Madrid: Fineo Editorial.

Escudero, J. M. (2002). La reforma de la reforma. ¿Qué calidad para quienes? Barcelona: Ariel.

La profesión docente en la sociedad del conocimiento. Una mirada a través de la revisión de investigaciones de los últimos 10 años. Lourdes Montero y Adriana Gewerc. Página 19 de 22 
Escudero, J. M. (2003). ¿Se puede mejorar la educación elevando los niveles de aprendizaje e implantando sistemas de rendición de cuentas? Profesorado. Revista de Curriculum y Formación del Profesorado, 7 (1-2), 49-56.

Esteve, J. M. (2006). La profesión docente en Europa: Perfil, tendencias y problemática. La formación inicial. Revista de Educación. [Monográfico sobre la Formación inicial del profesorado], 340, 19-40. Recuperado de http://www.revistaeducacion.mepsyd.es/re340/re340_03.pdf

Feiman-Nemser, S. y Buchman, M. (1986). The first year of teacher preparation: Transition to pedagogical thinking. Journal of Curriculum Studies, 18 (3), 239256. http://dx.doi.org/10.1080/0022027860180302

Fishman, B. \& Dede, Ch. (2016). ). Teaching and Technology: New Tools for New Times. In D. Gitomer \& C. Bell (Eds.) (2016). Handbook of Research on Teaching. 5th Edition (pp. 1269-1334). Washington DC: American Educational Research Association.

Flores, M. A. (2000). A Inducao no Ensino: Desafios e Constrangimentos. Lisboa: Instituto de Inovaçao Educacional.

Fraga-Varela, F. (2014). Procesos de construcción de creencias sobre Tecnología Educativa: estudio de casos en la formación inicial de Maestros de Educación. Primaria. (Tesis Doctoral). Universidade de Santiago de Compostela. Recuperado de http://dspace.usc.es/bitstream/10347/9831/1/rep_603.pdf

Fraga-Varela, F. y Rodríguez-Groba, A. (2016). Dilemas y desafíos de la tecnología educativa en el EEES: Percepciones y creencias de futuros maestros. Profesorado. Revista de Currículum y Formación de Profesorado 21 (1), 123142.

Gather Thurler, M. (2004). Innovar en el seno de la institución escolar. Barcelona: Graó.

Gewerc, A. y Alonso-Ferreiro, A. (2017). Influencias del plan de estudios del grado de maestro de primaria en el conocimiento profesional del profesorado.: El caso de la universidad de Santiago de Compostela. Profesorado: Revista de Curriculum y Formación del Profesorado, 21 (1), 39-58.

Gewerc, A. y Montero, L. (2013). Culturas, formación y desarrollo profesional. La integración de las TIC en las instituciones educativas. Revista de Educación, 362, 323-347.

Gewerc, A. y Montero, L. (2015). Conocimiento profesional y competencia digital en la formación del profesorado. El caso del Grado de Maestro en Educación Primaria. Revista Latinoamericana en Tecnología Educativa (RELATEC), 14 (1). http://relatec.unex.es/article/view/1829

González González, M. T. (1992). Centros escolares y cambio educativo. En J. LópezYáñez y J. M. Escudero Muñoz (coords.). Los desafíos de las Reformas Escolares: cambio educativo y formación para el cambio (pp. 71-96). Arquetipo Ediciones.

Goodson, I. \& Hargreaves, A. (2007). Series editors' preface. In Day, Ch.; Sammons, P.; Stobart, G.; Kington, A. \& Gu,Q. ( 2007). Teachers Matter. Conecting Lives, Work and Effectiveness (pp. xi-xv). Mc-Graw Hill Education: Berkshire (England).

Hargreaves, A. (1996). Profesorado, cultura y posmodernidad. Cambian los tiempos, cambian los profesores. Madrid: Morata.

Hargreaves, A. (2003). Enseñar en la sociedad del conocimiento. Barcelona: Octaedro.

La profesión docente en la sociedad del conocimiento. Una mirada a través de la revisión de investigaciones de los últimos 10 años. Lourdes Montero y Adriana Gewerc. Página 20 de 22 
Hauge, T. E. (2000). Student teachers' struggle in becoming professionals: Hopes and dilemmas in teacher education. In C. Day, A. \& Fernández, T. E. (Eds.). The Life and Work of Teachers. International Perspectives in Changing Times (pp. 157168). London: Routledge Falmer.

Hobson, A., Tomlinson, P. (2001). Secondary student teachers'preconceptions, experiences and evaluations of ITT: a UK study. Paper presented at The Annual Conference of the British Educational Research Association, University of Leeds, England, September 13-15.

Hudson, B.; Zgaga, P. \& Astrand, B. (Eds.). (2010). Advancing quality cultures for teacher education in Europe: Tensions and opportunities. Umea School of Education: University of Umea (Sweden).

Imbernón, F. (1998). La educación en el siglo XXI. Los retos del futuro inmediato. Barcelona: Graó.

Imbernón, F. (2017). Ser docente en una sociedad compleja. La difícil tarea de enseñar. Barcelona: Graó

Imbernón, F; Gimeno Sacristán, J., Rodríguez Martínez, C y Sureda, J. (2017). El profesorado, su formación y el trabajo educativo. Extraido de http://feae.eu/wpcontent/uploads/2017/11/F.-Imbern\%C3\%B3n-J.G.-Sacrist\%C3\%A1n-yotros.pdf

Innerarity, D. (2015). La sociedad del conocimiento nos hace más ignorantes. Cuadernos de Pedagogía, 452, 46-51.

Martínez Piñeiro, E. (2017). Desarrollo del conocimiento profesional a través del plan de estudios del grado de Maestro. El caso de la Universidad de Santiago de Compostela. En M $\mathrm{M}^{\mathrm{a}}$. T. Colén Riau (coord. y ed.). Retos y certezas sobre la construcción del conocimiento práctico en la formación de maestros. Una visión caleidoscópica (pp. 77-91). Barcelona: Octaedro ICE-UB.

Montero, L. (2006). Profesores y profesoras en un mundo cambiante: El papel clave de la formación inicial. Revista de Educación, 340, mayo-agosto, 66-86.

Montero, L. (2008). El retorno del interés por la formación inicial del profesorado. Monográficos Escuela, octubre, 4-6.

Montero, L.; Colén, M. y Martínez Piñeiro, E. (2017). Los estudios de Grado en la formación inicial de Maestros en Educación Primaria. Miradas de formadores y futuros maestros. Profesorado. Revista de Curriculum y Formación del Profesorado, 21 (1), 1-16. Monográfico: Desarrollo del conocimiento profesional en la formación inicial de maestros.

Montero, L. y Gewerc, A. (eds.) (2013). Una historia, cuatro historias. Acompañar proyectos de innovación educativa con las TIC. Barcelona: Graó.

Murillo, F.J. y Krichesky, G.J. (2015). Mejora de la Escuela: Medio siglo de lecciones aprendidas. REICE. Revista Iberoamericana sobre Calidad, Eficacia y Cambio en Educación, 13(1), 69-102.

Nóvoa, A. (2009). Profesores: ¿el futuro aún tardará mucho tiempo? En C. Vélaz de Medrano y D. Vaillant (Coords.). Aprendizaje y desarrollo profesional docente (pp. 49-55). Madrid: Organización de Estados Iberoamericanos para la Educación, la Ciencia y la Cultura (OEI).

Nóvoa, A. (2011). O regresso dos profesores. Pinhais: Melo.

Orden ECI/3857/2007 de 27 de diciembre de 2007, por la que se establecen los requisitos para la verificación de los títulos universitarios oficiales que habiliten para el ejercicio de la profesión de Maestro en Educación Primaria. Ministerio

La profesión docente en la sociedad del conocimiento. Una mirada a través de la revisión de investigaciones de los últimos 10 años. Lourdes Montero y Adriana Gewerc. Página 21 de 22 
de Educación. Recuperado de http://www.boe.es/boe/dias/2007/12/29/pdfs/A53747-53750.pdf

Organization for Economic Co-operation and Development (OECD). (2005). Teachers matter: Attracting, developing and retaining effective teachers. Paris, France: Author.

Sadin, E. (2017). La humanidad aumentada. La administración digital del mundo. Buenos Aires: Caja Negra Editores.

Schon, D. (1992). La formación de profesionales reflexivos. Hacia un nuevo diseño de la enseñanza y el aprendizaje en las profesiones. Madrid: Paidós/MEC.

Somekh, B. (2008). Factors affecting teachers' pedagogical adoption on ICT. In J. Voogt \& G. E. Kne-Zek (eds.). International Handbook of Information, Technology in Primary and Secondary Education (pp. 449-460). New York: Springer

Sosa, M ${ }^{\text {a }}$. J. y Valverde, J. (2017). Las macro-políticas educativas y el Proyecto de Educación Digital para la integración de las tecnologías desde la visión del profesorado. RED. Revista de Educación a Distancia, 53. Disponible en http://www.um.es/ead/red/53.

Subirats, M. (1999). La educación del siglo XXI: la urgencia de una educación moral. En F. Imbernón (coord.). La educación en el siglo XXI. Los retos del futuro inmediato (pp. 171-180). Barcelona: Graó

Stake, R.E. (1998). Investigación con estudio de casos. Madrid: Morata.

Torres Santomé, J, (2017). Políticas educativas y construcción de personalidades neoliberales y neocolonialistas. Madrid: Morata

Tyack, D. \& Tobin, W. (1994). The 'Grammar' of Schooling: Why Has it Been so Hard to Change? American Educational Research Journal, 31 (3), 453-479.

World Bank. (2010). Teacher politics around the world: Objectives rationale, methodological approach and products. Retrieved from http://siteresources.worldbank.org/EDUCATION/Resources/ Vegasetal_Teacherpoliciesaroundtheworld.draft.pdf

Wozney, L.; Venkatesh, V. \& Abrami, Ph. C. ( 2006). Implementing Computer technologies: Teachers' Perceptions and Practice. Journal of Technology and Teacher Education, 14 (1), 173-207.

Zapico, M ${ }^{\mathrm{a}}$ H.; Martínez Piñeiro, E. y Montero, L. (2017). ). Demandas sociales y formación inicial del profesorado: ¿Un callejón sin salida? Profesorado. Revista de Curriculum y Formación del Profesorado, 21 (1), 80-102.

Yin, R.K. (2003). Case study research. Design and methods. Thousand Oaks: Sage.

Zeichner, K. \& Conklin, H. (2008). Teacher Education Programs as sites for teachers preparation. In Cochran-Smith, M., Feiman-Nemser, S., \& McIntyre, D. J. (Eds.). Handbook of Research on Teacher Education. Enduring Questions in Changing Contexts. Third Edition (pp. 269-289). New York: Routledge

La profesión docente en la sociedad del conocimiento. Una mirada a través de la revisión de investigaciones de los últimos 10 años. Lourdes Montero y Adriana Gewerc. Página 22 de 22 